\title{
Length preserving multiresolution editing of curves
}

\author{
B. Sauvage S. Hahmann G.-P. Bonneau \\ Grenoble, France
}

\begin{abstract}
In this paper a method for multiresolution deformation of planar piecewise linear curves that preserves the curve length is presented. In a wavelet based multiresolution editing framework, the curve can be deformed at any level of resolution through its control points. Enforcing the length constraint is carried out in two steps. In a first step the multiresolution decomposition of the curve is used in order to approximate the initial curve length. In a second step the length constraint is satisfied exactly by iteratively smoothing the deformed curve. Wrinkle generation is an application the paper particularly focuses on. It is shown how the multiresolution definition of the curve allows to explicitly and intuitively control the scale of the generated wrinkles.
\end{abstract}

Keywords: multiresolution analysis, length constraint, deformation, curve length, linearization, wrinkle generation.

\section{Introduction}

Multiresolution analysis is widely used in various domains including visualization, geometric modeling and computer graphics. Multiresolution representations based on wavelets have been developed for parametric curves [3], and can be generalized to tensor-product surfaces, to surfaces of arbitrary topological type [13], to spherical data [17], and to volume data [4]. 
General multiresolution editing or deformation techniques for parametric curves have been explored in detail by Finkelstein and Salesin [8], Gortler and Cohen [9], Elber and Gotsman [6] using B-splines. Multiresolution curve editing methods with the constraint of constant enclosed area have been developed in $[5,11]$.

This paper introduces a multiresolution editing tool for piecewise linear planar curves which allows to satisfy the non-linear constraint of length preservation. Following traditional principles of animation [12], constant length deformation as well as constant volume or area, is generally required when modeling or animating deformable objects naturally. Beside general deformations through select-and-drag control points, the present paper focuses on a particular type of deformation: the generation of wrinkles in case of shrinking or compressing the curve.

The paper is organized as follows. In section 2 the basic mathematical tools are given. Section 3 presents a method of length preserving via optimization, based on a linearization of the length constraint. This method is discussed and improved in a two-step method presented in section 4. It is shown in particular how the generation of wrinkles is controlled in a whole editing process. Section 5 concludes and gives some possible future works.

\section{Tools}

Let us briefly sketch the notation of the wavelet based multiresolution analysis that will be used in this section. For more details see [14]. Suppose we have a certain functional space $E$ and some nested linear approximation spaces $V^{j} \subset E$ with $V^{0} \subset V^{1} \subset \cdots \subset V^{n}$. Let $V^{n}$ be of dimension $N$. Let $V^{j}$ be spanned by a basis of scaling functions $\varphi^{j}=\left[\varphi_{1}^{j}, \ldots, \varphi_{m}^{j}\right]^{T}$. A detail space $W^{j}$ is the complement of $V^{j}$ in $V^{j+1}$. Its basis of wavelets $\psi^{j}=\left[\psi_{1}^{j}, \ldots, \psi_{N-m}^{j}\right]^{T}$ is such that together with $\varphi^{j}$ they form a basis of $V^{j+1}$. The space $V^{n}$ can therefore be decomposed as follows:

$$
V^{n}=V^{n-1} \bigoplus W^{n-1}=V^{n-2} \bigoplus_{j=n-2}^{n-1} W^{j}=\cdots=V^{0} \bigoplus_{j=0}^{n-1} W^{j} .
$$

A multiresolution curve is then defined as a planar parametric curve $c(t)=$ $\left(c^{n}\right)^{T}\left(\varphi^{n}\right)$, element of $V^{n}$, where $c^{n}$ is a column of its $N$ control points in 
$\mathbb{R}^{2}$. Due to property (1) the same curve can be expressed at any level of resolution $e \in[0, n]$, i.e. as an element of $V_{e} \bigoplus_{j=e}^{n-1} W_{j}$. In terms of the basis functions, some coarse control points $c^{e}$ form approximations (global shape) of the initial control polygon and the detail coefficients $d^{e}, \ldots, d^{n-1}$ encode the character of the object:

$$
c(t)=\left(c^{e}\right)^{T}\left(\varphi^{e}\right)+\left(d^{e}\right)^{T}\left(\psi^{e}\right)+\cdots+\left(d^{n-1}\right)^{T}\left(\psi^{n-1}\right), \quad e=0, \ldots, n .
$$

The filter bank algorithm [14], [8] is used to compute the coefficients of all levels of resolutions from the initial coefficients $c^{n}$ and vice versa.

The length of a parametric curve $c(t)=(x(t), y(t))$ is given by $L=$ $\int \sqrt{x^{\prime}(t)^{2}+y^{\prime}(t)^{2}} d t$. The curves the present paper is dealing with are continuous and piecewise linear. In that case the length simplifies to $L=$ $\sum_{i=0}^{N-2}\left\|c_{i+1}-c_{i}\right\|_{2}$, where $c_{i}$ denote the control points at level $n$.

For the multiresolution representation of piecewise linear curves the scheme based on the Lazy wavelets [18] is used:

$$
\left\{\begin{array}{l}
c_{i}^{j}=c_{2 i}^{j+1} \\
d_{i}^{j}=c_{2 i+1}^{j+1}-\frac{1}{2}\left(c_{2 i}^{j+1}+c_{2 i+2}^{j+1}\right) .
\end{array}\right.
$$

The reasons for the particular choice of working only with piecewise linear curve are twofold. First, the length preserving deformation method of chapter 3 is intended to work dynamically. Linear curves in contrast to higher order polynomial curves simplify and accelerate the computations. Second, the particular effect of wrinkle creation in response to the motion of a control point is a purely visual effect. Therefore, on a computer screen no visual difference can be observed between displaying a dense polygonal curve or a rasterized polynomial curve.

In the case of polylines one can choose either to keep the total length constant or to preserve the length of each segment. We choose the second way because of two main reasons: first it ensures the balance between segment's length that is to say the control points don't gather in a small part of the curve. Secondly it allows the length constraints to be expressed in such a way that computationally inefficient square roots evaluations can be avoided.

The length constraints on a deformed curve $C$ with control points $c_{i}=\left(x_{i}, y_{i}\right)$ can now be reformulated as follows:

$$
f_{i}(C)=\Delta x_{i}^{2}+\Delta y_{i}^{2}-l_{i}^{2}=0 \quad \text { for } \quad i=0, \ldots, N-2,
$$


where $l_{i}$ is the reference length of segment $\left[c_{i} ; c_{i+1}\right]$ before deformation.

\section{Length preserving via optimization}

In a classical MR editing environment the user chooses a resolution level in which the curve is modified by displacing one coarse control point. The shape of the curve changes more or less locally depending on whether a low or a high resolution level has been chosen. The same procedure can be applied when integrating the additional constraint of keeping the curve length constant during deformation. We introduce here a method of length enforcing via optimization. Wrinkle generation is a particular application, which should occur naturally in the case of shrinking the curve or parts of it. The cost function to be minimize will take care of this feature and simultaneously produce smooth shapes. After presenting the basic ideas and the calculus we show some examples and discuss them.

\section{Overview}

Let $C_{R}$ be a given reference curve in $V^{n}$. Its length is denoted by $L_{R}$. Let $C_{A}$ be another curve in $V^{n}$ (resulting from the edition of $C_{R}$ ). The $(x, y)$-coordinate vectors of its control points are denoted by $\left(X_{A}, Y_{A}\right)$ and its length $L_{A}$ is assumed to be different from $L_{R}$. The problem to solve consists in length enforcing $C_{A}$, i.e. in finding a curve $C_{F}$ with control points $(X, Y)$ whose length is equal to $L_{R}$ and that is close to $C_{A}$. This is why $C_{A}$ is also named an attracting curve.

In the present context (see section 2), there are in total $N-1$ length constraints to satisfy and $N$ vector degrees of freedom available, that is to say $2 N$ scalar degrees of freedom. Hence a lot of length enforcing deformations would be possible. Let us illustrate in the following the use of a broad optimization method, i.e. minimization of a cost function which integrates a smoothness term and a distance term subject to the length constraints. The smoothness term prevents the resulting curve to have unwanted wiggles. The distance term is needed in order to respect the distance to the attracting curve $C_{A}$ as much as possible.

\section{Smoothness criterion}

In variational design a physical model is used for the description of a "smooth" 
or a "fair" curve or surface $[15,19,2]$. The most widely used fairness criterion originate from the observation that the shape of a thin elastic beam or a thin plate under deformation which minimizes the bending energy is always smooth, i.e. has a visual pleasing shape.

Since the bending energy for a planar parametric curve, $E=\int \kappa^{2}(t) d t$, is a non-linear functional, it is common to use instead the linearized version $[7,1]$ (both expressions are identical if $\left|c^{\prime}(t)\right| \equiv 1$ ):

$$
E=\int|c "(t)|^{2} d t=\int x "(t)^{2}+y "(t)^{2} d t
$$

In the case of a polyline, a discrete version is derived from a finite difference approximation of the derivatives:

$$
E(X, Y)=\sum_{i=1}^{N-2}\left\|\frac{1}{4}\left(c_{i-1}-2 c_{i}+c_{i+1}\right)\right\|^{2}=\frac{1}{2}\left(X^{T} H X+Y^{T} H Y\right)
$$

where

$$
H=\frac{1}{8}\left[\begin{array}{ccccccc}
1 & -2 & 1 & & & & \\
-2 & 5 & -4 & 1 & & 0 & \\
1 & -4 & 6 & -4 & 1 & & \\
& \ddots & \ddots & \ddots & \ddots & \ddots & \\
& & 1 & -4 & 6 & -4 & 1 \\
& 0 & & 1 & -4 & 5 & -2 \\
& & & & 1 & -2 & 1
\end{array}\right], \quad X=\left[\begin{array}{c}
x_{0} \\
x_{1} \\
\\
\vdots \\
x_{N-1}
\end{array}\right], \quad Y=\left[\begin{array}{c}
y_{0} \\
y_{1} \\
\vdots \\
\vdots \\
y_{N-1}
\end{array}\right] .
$$

\section{Length preserving deformation}

The flexibility of an energy based optimization can be enhanced by introducing an additional distance term: $D(X, Y)=\left\|X-X_{A}\right\|^{2}+\left\|Y-Y_{A}\right\|^{2}$.

The problem we aim to solve is now the following optimization problem:

$$
\min _{X, Y}\{(1-\beta) E(X, Y)+\beta D(X, Y)\} \quad \text { subject to } f_{i}=0, i=0, \ldots, N-2,
$$

where the constraints $f_{i}$ are defined in section 2 .

$0 \leq \beta \leq 1$ is a scalar value which balances between a smoother curve and a curve closer to $C_{A}$. 
The length constraints are quadratic expressions. Since minimizing a quadratic cost function subject to quadratic constraints is costly and since one of our objectives is to provide a fast algorithm, let us approach the length constraint by using linearized constraints instead.

\section{Linearizing the length constraints}

With the technique of Lagrange multipliers [10], the minimization problem is restated to finding a stationary point of the following function with respect to $X, Y$ and $\Lambda$ :

$$
(1-\beta) E(X, Y)+\beta D(X, Y)+\sum_{i=0}^{N-2} \lambda_{i} f_{i}
$$

where $\Lambda=\left(\lambda_{0}, \ldots, \lambda_{N-2}\right)^{T}$ is the vector of Lagrange multipliers.

In order to linearize each $f_{i}(X, Y)=\left(\Delta x_{i}\right)^{2}+\left(\Delta y_{i}\right)^{2}-l_{i}$, let us approach it by the linear part $\tilde{f}_{i}$ of its Taylor expansion with respect to $\Delta x_{i}$ and $\Delta y_{i}$ in the neighborhood of $\Delta x_{i}^{A}$ and $\Delta y_{i}^{A}$. Hence one gets

$$
\tilde{f}_{i}=2 \Delta x_{i}^{A} \Delta x_{i}-\left(\Delta x_{i}^{A}\right)^{2}+2 \Delta y_{i}^{A} \Delta y_{i}-\left(\Delta y_{i}^{A}\right)^{2}-l_{i}^{2} .
$$

Remark: In order to replace $f_{i}$ by the linearized expression $\tilde{f}_{i}$ we have to ensure that $\Delta x_{i}, \Delta y_{i}$ are close to $\Delta x_{i}^{A}, \Delta y_{i}^{A}$. In other words the curve $C_{A}$ must nearly satisfy the length constraints.

Assuming that the approximation of $f_{i}$ by $\tilde{f}_{i}$ holds, the problem can be restated as solving:

$$
\vec{\nabla} g=0 \quad \text { where } \quad g(X, Y, \Lambda)=(1-\beta) E(X, Y)+\beta D(X, Y)+\sum \lambda_{i} \tilde{f}_{i} .
$$

A symmetric square sparse system of linear equations has to be solved:

$$
\left\{\begin{array}{l}
(1-\beta) H X+2 \beta X+2 \Delta_{X} \Lambda=2 \beta X_{A} \\
(1-\beta) H Y+2 \beta Y+2 \Delta_{Y} \Lambda=2 \beta Y_{A} \\
2 \Delta_{X}^{T} X+2 \Delta_{Y}^{T} Y=b
\end{array}\right.
$$

where where $b=\left(l_{i}^{2}+\left(\Delta x_{i}^{A}\right)^{2}+\left(\Delta y_{i}^{A}\right)^{2}\right)_{i=0 \ldots N-2}^{T}$, and where $\Delta_{X}$ (resp. $\Delta_{Y}$ with $y$ instead of $x)$ is a $N \times(N-1)$ matrix: 


$$
\Delta_{X}=\left[\begin{array}{ccccc}
-\Delta x_{0}^{A} & & & & \\
\Delta x_{0}^{A} & -\Delta x_{1}^{A} & & 0 & \\
& \Delta x_{1}^{A} & \ddots & & \\
& & \ddots & \ddots & \\
& 0 & & \ddots & -\Delta x_{N-2}^{A} \\
& & & & \Delta x_{N-2}^{A}
\end{array}\right] .
$$

Let us note that due to the linearization the result $(X, Y)$ is not exact for the length constraints. In order to increase the precision, the solving can be encapsulated in a loop which iterates the system solving.

\section{Examples and discussion}

Figure 1 illustrates the role of $\beta$ : a deformation is applied to the initial curve by editing it at the coarsest level of resolution. Fig. 1(a) shows the initial curve together with its coarsest approximation consisting of 2 control points. Figure 1(b) shows the deformed coarse polygon and the corresponding reconstructed curve without length preservation. This is the attracting curve $C_{A}$. The problem is then solved for different values of $\beta$, see figures 1(c), 1(d), and 1(e). As expected, the role of $\beta$ is to control the shape of the resulting curve. A small value gives preference to a smooth curve in the sense of minimal bending energy. A higher value keeps the mean distance to $C_{A}$ (gray) as small as possible. In the particular case of shrinking the initial curve, as illustrated in that figure, $\beta$ controls the creation of wrinkles. A high value produces small and frequent wrinkles, while a small value stands for larger and less frequent wrinkle.

These results suggest that this method could be used as it is, because it allows the curve to be deformed at a given scale (controlled through the value of $\beta$ ) while preserving its length. But two reasons make it impractical to use:

- If the edited curve $C_{A}$ is far from satisfying the length constraint, then the error due to linearization is important. We hence have to iterate the solving in order to bound the error below some threshold which may be expensive (tens of solvings are necessary for fig. 1).

- As shown in Figure 1, $\beta$ controls the appearance of the curve. However the choice of $\beta$ must take into account a lot of parameters including the scale of the wrinkles and the number of control points. Hence it 


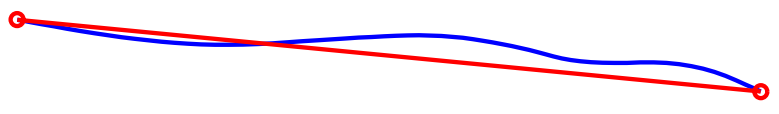

(a) initial curve

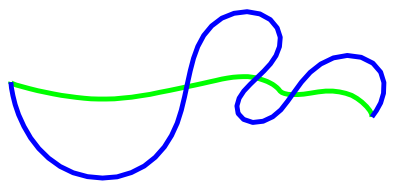

(c) $\beta=0.001$

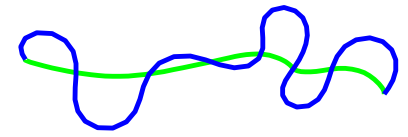

(d) $\beta=0.01$

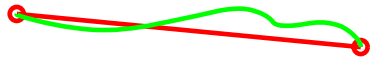

(b) edited curve without length preservation

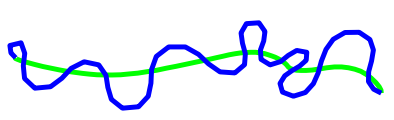

(e) $\beta=0.03$

Figure 1: Role of $\beta$ : the initial curve (a) with 129 control points is edited at the coarsest level (b) (in gray : reconstructed attracting curve). In (c), (d) and (e) : 3 final length preserving curves (in black) are given for different values of $\beta$.

is quite impractical to choose the wrinkling scale. We aim to have an easy-to-use editing tool.

However, if the attracting curve nearly satisfies the length constraint, this optimization method is very effective in precisely meeting the prescribed length.

\section{Control of wrinkle generation}

In this section we introduce a new step into the editing process taking place before the optimization method presented in the previous section. In order to compensate for the drawbacks of the optimization, it aims to compute in linear time an attracting curve suitable for the optimization step because it sufficiently approximates the lengths constraints while the scale of the deformations induced by these constraints can be explicitly and intuitively controlled by the user.

The main idea is to modify the details of the edited curve at an intermediate level $e<=w<n$ so that the control polygon at level $w+1$ has the 
same length as the initial coarse polygon. Hence this step is called explicit length preserving. An important advantage of modifying details is that the attractive curve built in this way is close to the edited curve because their coarse control polygons at level $w$ match whenever possible. That is to say this curve follows as much as possible the modification applied by the user.

\subsection{Editing loop with explicit length preservation step}

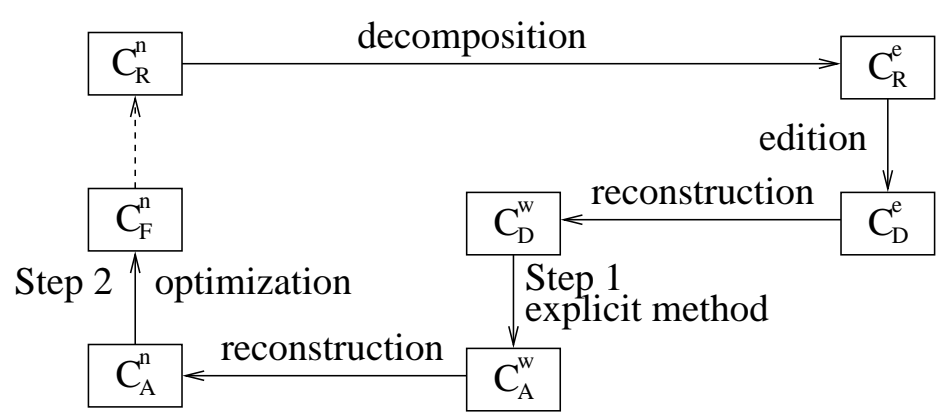

Figure 2: Enhanced editing loop for wrinkle generation

Let $C_{R}(t) \in V^{n}$ be the initial planar piecewise linear curve the user wants to edit while keeping its length constant. Two resolution levels may be specified by the user: level $e$, at which the user will edit the control polygon, and level $w$, at which wrinkles will be generated if required by the length constraint. The editing process follows the graph of Figure 2. Each of the six arrows corresponds to a particular action described in the following paragraphs and illustrated by a real example in Figure 3 :

Decomposition: The curve is decomposed into $C_{R}^{e}$ at the level $e$, chosen by the user (see fig. 3(a)). The superscripts denote the level of resolution.

Edition: The coarse control polygon $C_{R}^{e}$ a level $e$ is modified by the user. In Fig. 3(b) the user "pinched" the right part of the curve. The corresponding deformed curve $C_{D}$ (doted line in fig $3(\mathrm{~b})$ ) has not the same length as the reference curve $C_{R}$. Notice that the choice of level $e$ determines the extent of the deformation.

Reconstruction: The deformed curve is then partially reconstructed at level $w(e \leq w<n)$, chosen by the user (in fig. 3(c): $w=e+1$ ).

Explicit length preserving: An attracting curve $C_{A}$ is constructed (see fig. $3(\mathrm{~d})$ ). This curve is obtained by modifying $C_{D}^{w}$ using the details gradually 
along the control polygon in order to obtain a control polygon $C_{A}^{w+1}$ whose edges have the same length as the control polygon segments of $C_{R}$ at level $w+1$. Some control points at level $w$ may be modified as well in case of stretching. More details can be found in [16]. Modifying details at level $w$ is equivalent to modifying corresponding control points at level $w+1$. In the example they are highlighted as dark dots in fig. 3(c). The choice of $w>e$ for length approximation increases the number of coarse control points defining the same portion of the curve and hence determines the number of wrinkles on this portion. The closer to the highest level $n$ is $w$ the higher is the frequency of the wrinkles.

Reconstruction: The attractive curve is then completely reconstructed (fig. 3(d)) by re-inserting the details of $C_{R}$ at the levels $w+1, \ldots, n$. After reconstruction, the length of $C_{A}$ is close to satisfy the length constraint thanks to the length preservation at level $w+1$. Note, that the use of a lazy wavelet scheme leads to some sharp features in the reconstructed curve.

Length preserving by smoothing: The optimization method (see section 3) applied to $C_{A}$ leads to the final curve $C_{F}$ (fig. $3(\mathrm{e})$ ). It precisely satisfies the length constraints, has a smooth shape, and it is close to the attracting curve $C_{A}$. The main role of this optimization step remains now to smooth the curve. Figure 3(d) illustrates the effect of the smoothing, whose cost dramatically drops.

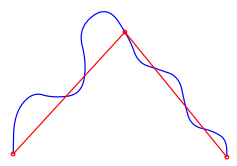

(a) $C_{R}$ and $C_{R}^{e}$

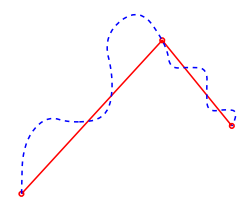

(b) $C_{D}$ and

$C_{D}^{e}$

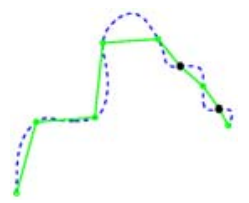

(c) $C_{D}$ and $C_{D}^{w+1}$

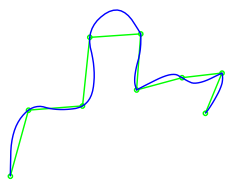

(d) $C_{A}$ and $C_{A}^{w+1}$

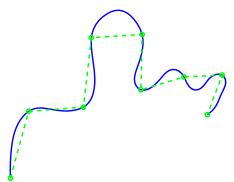

(e) $C_{F}$ and $C_{F}^{w+1}$

Figure 3: The editing loop. The curves decomposed at the level $e$ or $w$ are represented by their control polygon.

\subsection{Results}

We present here an example of MR editing that illustrates the important points we focused on. 


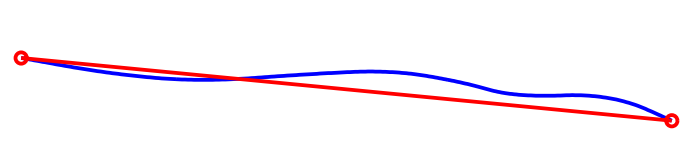

(a) Initial curve and its control polygon.

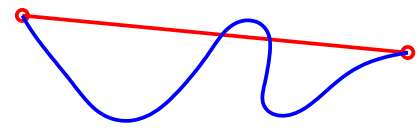

(b) First deformation (one editing loop).

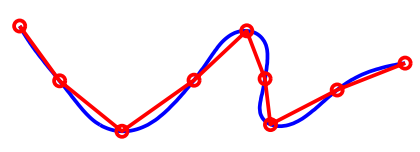

(c) First deformation.

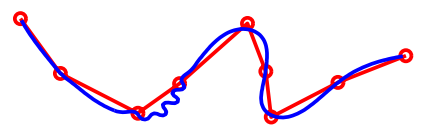

(d) Second deformation.

Figure 4: MR editing: the initial curve (a) is at first edited at the coarsest level (b) and then at an intermediate level (c) and (d).

The Figure 4 shows two successive deformations at different levels of decomposition (of the same curve as fig. 1), i.e. the result of two editing loops. The initial curve 4(a) with 129 control points is edited at the coarsest level $e=0$ and its length is adapted at the scale $w=1$. Hence it provides large wrinkles $(4(\mathrm{~b}))$. Then it is edited at the scale $e=3$ (fig. 4(c)): 2 neighboring control points are moved closer. Since the length is preserved at the scale $w=6$, small wrinkles are created (fig. 4(d)). Let us note that the curve is modified locally. We also see that multiresolution provides a flexible framework for curve editing. The levels $e$ and $w$ can be chosen in order to get exactly the deformation at the scale you want and on the extent you want.

The whole algorithm works dynamically, i.e. the user selects-and-drags a coarse control point immediately the length preserving curve is displayed. In fact, the first step has linear complexity and the second step needs generally no more than 5 linear system solvings. It is implemented with sparse matrices efficient conjugate gradient methods for reducing the computing time. As an example, the deformations of the curve in figure 4 are obtained in less than some tens ms each. 


\section{Conclusion}

A method for length preserving multiresolution editing of planar curves has been introduced. The method combines an explicit length preserving step together with an optimization step in order to provide smooth results with an explicit and intuitive control of the scale of the deformations.

Current and future research concerns the integration of other non-linear constraints into a multiresolution editing environment and the generalization to space curves and surfaces.

\section{References}

[1] Bonneau, G.-P., And Hagen, H. Variational design of rational bézier curves and surfaces. Curves and Surfaces in Geometric Design (1994), $51-58$.

[2] Celniker, G., And Gossard, D. Deformable curve and surface finite-elements for free-form shape design. ACM SIGGRAPH Computer Graphics 25, 4 (1991), 257-266.

[3] Chui, C., And QuAK, E. Wavelets on a bounded interval. In $\mathrm{Nu}^{-}$ merical Methods of Approximation Theory (1992), D. Braess and L. L. Schumaker, Eds., Birkhäuser Verlag, Basel, pp. 1-24.

[4] Cignoni, P., Montani, C., Puppo, E., and Scopigno, R. Multiresolution representation and visualization of volume data. IEEE Transactions on Visualization and Computer Graphics 3, 4 (1997), 352369.

[5] Elber, G. Multiresolution curve editing with linear constraints. In 6th ACM/IEEE Symposium on Solid Modeling and Applications (June 2001), Ann Arbor, Michigan, pp. 109-119.

[6] Elber, G., And Gotsman, C. Multiresolution control for nonuniform bspline curve editing. In The third Pacific Graphics Conference on Computer Graphics and Applications, Seoul, Korea (1995), pp. 267-278.

[7] Farin, G., Rein, G., Sapidis, N., and Worsey, A. Fairing cubic b-spline curves. Computer Aided Geometric Design 4 (1987), 91-103. 
[8] Finkelstein, A., And Salesin, D. H. Multiresolution curves. Computer Graphics 28 (1994), 261-268.

[9] Gortler, S., And Cohen, M. Hierarchical and variational geometric modeling with wavelets. Proceedings of the 1995 symposium on Interactive 3 D graphics (1995), 35-.

[10] Gottfried, B. S., And Weisman, J. Introduction to Optimization Theory. Prentice Hall, Englewood Cliffs, New Jersey, 1973.

[11] Hahmann, S., Bonneau, G., And Sauvage, B. Area preserving deformation of multiresolution curves. Submitted (2003).

[12] Lasseter, J. Principles of traditional animation applied to 3d computer animation. In Computer Graphics Proceedings (SIGGRAPH 87) (1987), pp. $35-44$.

[13] Lounsbery, M., DeRose, T., And Warren, J. Multiresolution analysis for surfaces of arbitrary topological type. ACM Transaction on Graphics 16, 1 (1997), 34-73.

[14] Mallat, S. A theory for multiresolution signal decomposition: the wavelet representation. IEEE Transactions on Pattern Analysis and Machine Intelligence 11 (1989), 674-693.

[15] Nowacki, H., And Reese, D. Design and fairing ship surfaces. Surfaces in CAGD (1983), 121-134.

[16] Sauvage, B. Déformartion de courbes multirésolution sous contraintes. Mémoire de DEA, Université Joseph Fourier, Juin 2002.

[17] Schröder, P., And Sweldens, W. Spherical wavelets: efficiently representing functions on the sphere. In Computer Graphics Proceedings (SIGGRAPH 95) (1995), pp. 161-172.

[18] Sweldens, W. The lifting scheme: A construction of second generation wavelets. SIAM J. Math. Anal. 29, 2 (1997), 511-546.

[19] Welch, W., And Witkin, A. Variational surface modeling. In ACM SIGGRAPH Computer Graphics (1992), pp. 157-166. 


\section{Basile Sauvage}

Laboratoire LMC-IMAG, BP.53

F-38041 Grenoble cedex 9, France

email: Basile.Sauvage@imag.fr

\section{Stefanie Hahmann}

Laboratoire LMC-IMAG, BP.53

F-38041 Grenoble cedex 9, France email: Stefanie.Hahmann@imag.fr

\section{Georges-Pierre Bonneau}

Laboratoire EVASION-GRAVIR-IMAG

INRIA Rhône-Alpes

655, avenue de l'Europe

F-38330 Montbonnot, France

email: Georges-Pierre.Bonneau@imag.fr 